\title{
Mucocele de apêndice
}

\section{Mucocele of the appendix}

\author{
Roberto Gibson Ferreira Costa, ACBC-BA ${ }^{1}$
}

\section{INTRODUÇÃO}

A mucocele de apêndice é um termo inicialmente descrito por Rokitansky em 1842 e formalmente denominado por Feren em 1876, que define o aumento do apêndice cecal pela produção e acúmulo intraluminar de muco, devido a alterações da camada mucosa e muscular. Em 1940 Woodruff e Mc Donald determinaram a classificação das mucoceles em benignas e malignas. É uma entidade rara e geralmente tem seu diagnóstico feito no período intra-operatório'.

A mucocele apendicular pode ser provocada por quatro tipos de processos patológicos distintos.

Tipo l - Obstrução da comunicação cecoapendicular por fecalito ou retração cicatricial, que se denomina mucocele simples do apêndice ou cisto de retenção;

Tipo II - Hiperplasia mucosa focal ou difusa sem atipia celular da mucosa do apêndice (pólipos hiperplásticos);

Tipo III - Cistoadenoma mucinoso com certo grau de atipia celular;

Tipo IV - Cistoadenocarcinoma mucinoso.

Os processos patológicos do Tipo I e ll, são considerados benignos e o Tipo III de transição. O Tipo IV é uma neoplasia maligna, que a disseminação de células epiteliais no interior da cavidade abdominal ocorre em $6 \%$ dos casos e se denomina pseudomixoma peritoneal.

O objetivo desta publicação é apresentar um caso de mucocele de apêndice cujo diagnóstico foi feito no período intra-operatório e fazer uma breve revisão da literatura.

\section{RELATO DO CASO}

Paciente do gênero masculino, pardo, 49 anos, deu entrada no Serviço de Gastroenterologia com queixa de vários episódios de epigastralgia, vômitos, plenitude gástrica e pirose, sendo submetido a endoscopia digestiva alta que revelou deformidade bulbar associada à úlcera duodenal ativa com Hpylori (+) e esofagite erosiva grau I.
Após seis meses do tratamento clínico, o paciente retornou ao ambulatório de gastroenterologia com recidiva da sintomatologia, sendo submetido a nova endoscopia que revelou, estenose de piloro, deformidade bulbar e gastrite moderada.

Diante disso o paciente foi encaminhado ao serviço de cirurgia para avaliação e tratamento cirúrgico onde foi submetido, após exames pré-operatórios, a gastrectomia subtotal com reconstrução a Y de Roux.

Durante a inspeção da cavidade, foi encontrado o apêndice cecal de aspecto cístico, bastante aumentado de volume, discretamente aderido ao ceco e base livre de aproximadamente $1 \mathrm{~cm}$ de diâmetro.

Após nova inspeção detalhada da cavidade e não sendo encontrado nenhuma alteração ou sinais de implante de mucina, foi feita a proteção da parede abdominal, exposição do ceco e apêndice para fora da cavidade e realizada a apendicectomia, segundo a técnica padronizada, devido a não existência de infiltração tumoral, linfonodomegalia ou qualquer tipo de comprometimento na base do apêndice, (Figura 1).

O paciente evoluiu bem na enfermaria sem queixas e sem intercorrência obtendo alta hospitalar no $7^{\circ}$ dia de pós-operatório.

O exame macroscópico, da peça cirúrgica, revelou um apêndice cecal dilatado, medindo 9,5 ×4 cm, superfície externa acastanhada, lisa, brilhante, observandose dilatação cística preenchida por conteúdo brancacento e de aspecto mucóide. A microscopia mostra que se trata

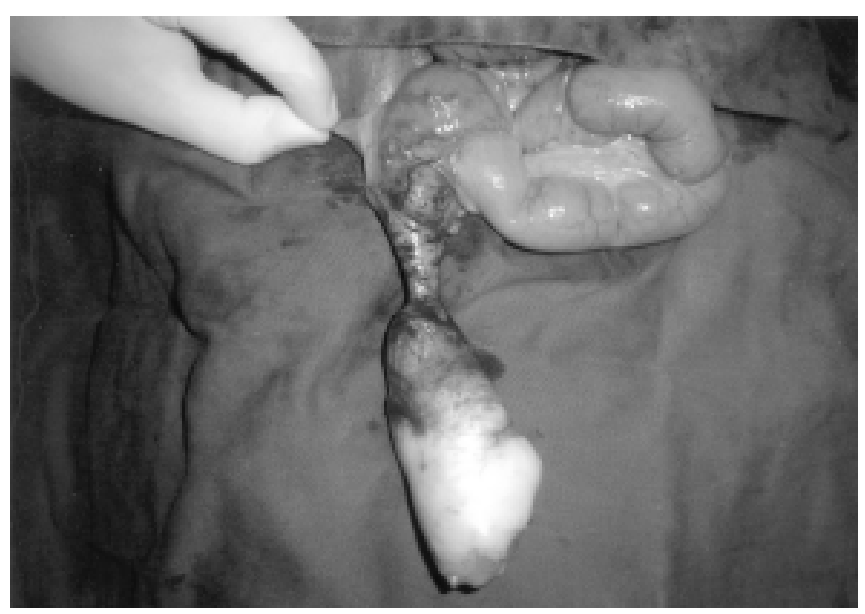

Figura 1 - Exteriorização da mucocele de apêndice, ceco e íleo terminal.

Trabalho realizado no Hospital Senhor do Bonfim - Xiquexique- BA.

1. Chefe do Serviço de Cirurgia Geral do Hospital Senhor do Bonfim - Xiquexique - BA. 
de cistoadenoma mucinoso do apêndice cecal sem evidências de malignidade histológica (Figura 2).

\section{DISCUSSÃO}

Do ponto de vista epidemiológico, a mucocele do apêndice aumenta a sua incidência a partir da sexta década de vida observando sua maior freqüência em muIheres, aparecendo em 0,1 a $0,4 \%$ de todas as apendicectomias e $0,15 \%$ em achados de necropsia ${ }^{1}$, sendo o cistoadenoma mucinoso a causa mais freqüente da mucocele apendicular representando $50 \%$ dos casos. A mucocele pode estar associada a outras neoplasias como o adenocarcinoma do cólon entre 0 a $21 \%$ e dos ovários entre 4 a $24 \%{ }^{1,4}$.

Os sinais e sintomas são inespecíficos e quando surgem são: discreta dor em fossa ilíaca direita aguda ou crônica, presença de massa palpável, alterações do trânsito intestinal, anemia, hematoquesia, enterorragia, insuficiência renal obstrutiva, hematúria e a depender da localização do apêndice pode ocorrer outros sinais e sintomas ${ }^{1,4}$. Devido a isso a mucocele de apêndice é, mais freqüentemente, encontrada acidentalmente durante a operação, como no caso aqui relatado, ou em uma exploração radiológica ou procedimento laparoscópico em 23 a 50\% dos casos².

Os achados laboratoriais são inespecíficos, sendo nos casos em que a mucocele tenha como causa o cistoadenocarcinoma pode ocorrer aumento significativo dos níveis de marcadores tumorais, como CEA e o CA50, tanto no pré-operatório como no pós-operatório ${ }^{5}$. Já a ultrassonografia, o enema baritado e a tomografia computadorizada podem e devem ser usados na suspeita diagnóstica de mucocele de apêndice ${ }^{1,2}$.

A confirmação diagnostica pré-operatório pela biopsia percutânea é controversa, devido ao risco de disseminação de células neoplasicas no trajeto da punção ou no peritônio, o que poderá comprometer totalmente a possibilidade de cura ${ }^{5}$.

Apesar da grande dificuldade, é muito importante o diagnóstico pré-operatório, na presença de forte suspeita diagnóstica de mucocele, para que se possa manipular cuidadosamente a lesão no período intra-operatório, evitando o rompimento da mesma e a disseminação de células tumorais no peritônio, que levam ao desenvolvimento do Pseudomixoma peritonial, que é a complicação mais temível desta patologia, pois alem de ter difícil tratamento por envolver cirurgia radical de peritônio, associado a quimioterapia intraperitonial e sistêmica, com baixo índice de cura, tem péssimo prognostico ${ }^{1,2,5}$.

Em relação a sobrevida dos pacientes, a literatura publica os seguintes dados:

Cistoadenoma mucinoso de apêndice - 91 a $100 \%$ em cinco anos ${ }^{1}$, independente de ocorrer perfuração ou não da lesão.

Cistoadenocarcinoma que desenvolvem o pseudomixoma peritoneal por disseminação celular - 25\% em 5 anos.

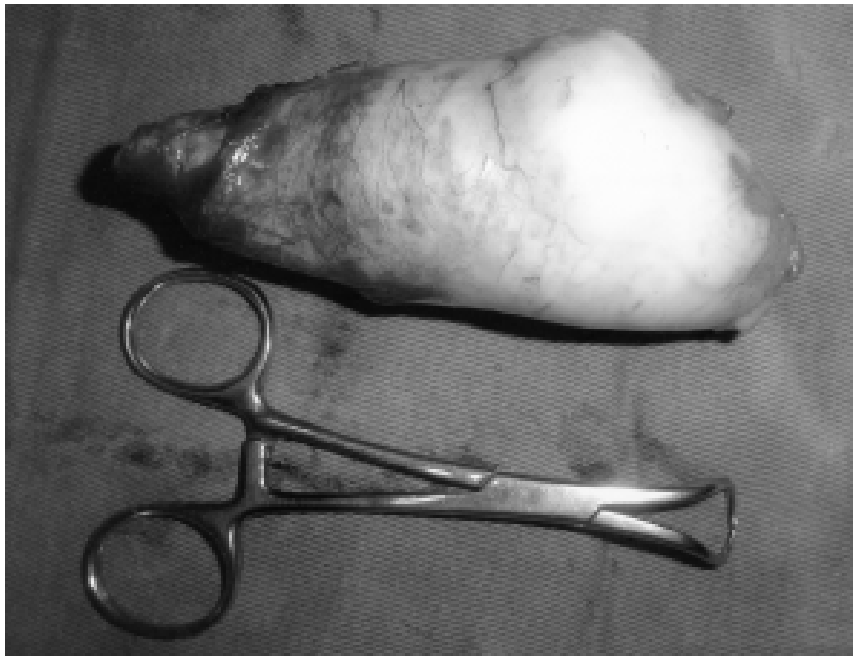

Figura 2 - Mucocele do apêndice cecal.

No período de 1976 a 2000, foi feita uma revisão de 135 casos de mucocele de apêndice, tratados na Mayo Clinic e se constatou que $1 / 3$ dos casos eram de cistadenocarcinoma mucinoso $0^{5}$. Também se observou que a maioria dos casos com sintomatologia exuberante no préoperatório eram portadores do carcinoma e que lesões maiores de $2 \mathrm{~cm}$, devem ser removidas cirurgicamente, devido ao maior risco de malignidade ${ }^{5}$. Ainda de acordo com este estudo, verificou-se que nos trabalhos mais antigos e nos primeiros pacientes, optava-se por uma simples apendicectomia nos casos suspeitos de mucocele, obtendo-se resultados satisfatórios quando se tratava de cistoadenoma, mas quando o exame histopatologico mostrava malignidade, o paciente era submetido a uma nova intervenção para uma ressecção radical da região acometida, aumentando a morbidade e mortalidade, e consequentemente o risco de disseminação neoplasia no peritônio ${ }^{1,5}$. Portanto o tratamento inicial padrão para a mucocele de apêndice, passou a ser a hemicolectomia direita ${ }^{1,5}$, mas alguns autores autorizam apenas a apendicectomia como aceitável para tratamento de tumores benignos ${ }^{1}$.

A hemicolectomia direita videolaparoscopica pode ser realizada, porem deve-se tomar bastante cuidado na manipulação da peça com as pinças, para evitar a ruptura do apêndice, que se encontra friável e dilatado nesta patologia 1,5. A apendicectomia videolaparoscopica é contra indicada, devido ao alto risco de disseminação neoplasica, pela manipulação direta da peça com as pinças, que aumenta muito a possibilidade de ruptura do apêndice durante o procedimento ${ }^{1,5}$.

No caso aqui relatado, como se tratava de um apêndice com base livre de neoplasia, sem sinais de implante tumoral ou linfonodomegalia regional e como foi um achado acidental durante uma operação de grande porte, optou-se pela apendicectomia como menos traumática e mais adequada naquele momento para o paciente em questão.

Este caso comprova o que vem sendo relatado na literatura e em trabalhos publicados, que realmente a maioria dos diagnósticos de mucocele 
apendicular ocorrem acidentalmente durante uma operação, principalmente pela pobreza de sinais e sintomas que nos leve a sua suspeição. Apesar disso, devemos manter em mente a possibilidade da mucocele de apêndice, na presença de dor em fossa ilíaca direita associada ou não a vários sinais e sintomas por mais discretos que sejam e que venham a surgir agudamente ou se tornem crônicos.

\title{
A B S T R A C T
}

\begin{abstract}
Appendiceal mucocele is a rare entity characterized by a gross enlargement of the appendix from accumulation of mucoid substance within the lumen. It is encountered in only $0.1-0.4 \%$ of all appendicectomies with a female predominance (M/F: 1/4) and a mean age of more than 50 years at the time of presentation. Because of that, appendiceal mucocele is often incidentally discovered either during surgery or on radiologic examination. A case of benign appendiceal mucocele is reported here, in a 49 years old male. The pathogenesis and the different surgical strategies are discussed.
\end{abstract}

Key words: Mucocele. Appendix. Cystadenoma, mucinous.

\section{REFERENCIAS}

1. Haritopoulos KN, Brown DC, Lewis P, Mansour F, Eltayar AR, Labruzzo $C$ et al. Appendiceal mucocoele: a case report and rewiew of the literature. Int Surg. 2001; 86(4):256-62.

2. Kestering DM, Araujo PA, Andriani AC, Kruel NF, Gonçalves WM. Mucocele de apêndice e pseudomixoma peritoneal. Rev Col Bras Cir. 1997; 24(6):441-2.

3. Emmi S, Gallasso MG, Ursino V, Scala R, Guastella T. [Appendicial mucoceles. A case report]. Minerva Chir. 1998; 53(10):807-10.

4. Pereira JCR, Silva Jr CA. Mucocele do apêndice. Relato de caso. Rev Col Bras Cir. 2000; 28(3):225-7.

5. Yamene YD, Yamane H, Castro Jr PC, Marsilic A, Mesquita RB, Lopes PF. Mucocele de apêndice. Relato de caso e Revisão de Literatura. Rev Bras Coloproct. 2005; 25(3):256-60.
Recebido em 15/03/2006

Aceito para publicação em 19/05/2006

Conflito de interesse: nenhum

Fonte de financiamento: nenhuma

\section{Como citar este artigo:}

Costa RGF. Mucocele de apêndice. Rev Col Bras Cir. [periódico na Internet] 2009; 36(2). Disponível em URL: http://www.scielo.br/rcbc

\section{Endereço para correspondência:}

Roberto Gibson F. Costa

E-mail: rrgibson@terra.com.br 